\title{
MEASURING SIMILARITY BETWEEN MOBILITY MODELS AND REAL WORLD MOTION TRAJECTORIES
}

\author{
Morteza Mousavi Barroudi
}

Student Member, IEEE, Aaron Harwood, Shanika Karunasekera

\begin{abstract}
Various mobility models have been proposed to represent the motion behaviour of mobile nodes in the real world. Selection of the most similar mobility model to a given real world environment is a challenging issue which has a significant impact on the quality of performance evaluation of different network protocols. In this paper we propose a methodology for measurement of similarity between mobility models used in mobile networks simulation and real world mobility scenarios with different transportation modes. We explain our mobility metrics we have used for analysis of motion behavior of mobile nodes and a pre-processing method which makes our trajectories suitable for extraction and calculation of these metrics considering shape of the road networks and GPS noise. Then we use a feature selection method to find the most discriminative features which are able to distinguish between trajectories with different transportation modes using a supervised learning and feature ranking method. Subsequently, using our selected feature space we perform Fuzzy C-means Clustering to find the degree of similarity between each of our mobility models and real world trajectories with different transportation modes. Our methodology can be used to select the most similar mobility model suitable for simulation of mobile network protocols (such as DTN and MANETs protocols) in a particular real world area.
\end{abstract}

\section{KEYWORDS}

Mobility Models, Similarity Analysis, Transportation Modes, Mobile Networks

\section{INTRODUCTION}

Researchers often use simulators such as Glomosim, Opnet, NS2, and OMNET++ to simulate their mobile networks protocols. These simulators provide facilities for simulation of motion of mobile nodes in the plain simulation area using mobility models such as Randowm Waypoint, Random Walk, Brownian Motion [1], Markovian [2], and RPGM [3], [4], [2]. One question here is which of these highly used mobility models perform more similar to real world motion scenarios? To be able to find the best mobility models for simulation and performance evaluation of mobile network protocols we need to perform similarity analysis between mobility models and real world motion behaviour of mobile nodes in the particular real world area in which we are going to implement our networking protocol.

Natarajan Meghanathan et al. (Eds) : WiMONe, NCS, SPM, CSEIT - 2014

pp. 43-55, 2014. (C) CS \& IT-CSCP 2014

DOI : $10.5121 /$ csit.2014.41205 
Research works such as [3], [4] and [5] proposed mobility models for simulation of mobile node motion in mobile

Morteza Mousavi Barroudi is with NICTA VRL, Department of CIS, The University of Melbourne, Melbourne, Australia. email: mousavi@student.unimelb.edu.au.

Aaron Harwood is with NICTA VRL, Department of CIS, The University of Melbourne, Melbourne, Australia. email: aharwood@unimelb.edu.au.

Shanika Karunasekera is with NICTA VRL, Department of CIS, The University of Melbourne, Melbourne, Australia. email: karus@unimelb.edu.au.

National ICT Australia (NICTA) is funded by the Australian Government's Backing Australia's Ability initiative, in part through the Australian Research Council. environments. However, they did not provide a comprehensive methodology for analysis of the similarity of their proposed mobility models and real world scenarios with different transportation modes, ("Bike", "Car", "Train", "Walk", "Bus").

In this paper we extend the methodology we introduced in our previous work [6] to propose a comprehensive methodology for measuring the similarity between real world movement scenarios with different transportation modes and mobility models. Aiming that we perform a feature selection method to find the most discriminative feature sets which are able to classify different trajectories with different transportation modes into their right classes with optimal set cardinality [6]. Subsequently, we propose a similarity measurement method which is able to calculate the degree of similarity between mobility models and real world trajectories with different transportation modes.

Before performing the similarity analysis method, we need to pre-process our data to make it suitable for feature extraction. We use our proposed method in [7] to interpolate missing waypoints (up-sampling) and reduce GPS noise to have regular and reliable real world trajectories. Finally, to calculate the degree of similarity between mobility models and real world transportation modes, we use Fuzzy C-means Clustering (FCM) [8]. This clustering method calculates the degree of membership of each sample in the feature space to each of the clusters. We use this fuzzy membership as a measure for the similarity between our samples (trajectories with different transportation modes) and clusters of mobility models. Before, performing the clustering we extract features from 5 classes of mobility models, (Random Waypoint (RWP), Random Walk (RW), Levy Walk(LW), Manhattan (MAN), and RPGM [6], [3], [4], [2], [5]) as representatives of mobility models. We also extract the features of our transportation mode trajectories. Then we use the optimal and the most discriminative feature set calculated in the feature selection phase as the feature space in our FCM clustering and consider the estimated degrees of membership as the degree of similarity between each of the transportation modes and each of the mobility models.

The remaining of the paper is organized as follows. In section II, we shortly discuss the most related research works to ours. In Section III, we briefly introduce our mobility metrics. Then we introduce our proposed trajectory pre-processing, classification and feature selection methods in IV-B. IV-D introduces our similarity analysis method using fuzzy Cmeans clustering. Finally, Section V contains the conclusion and future work. 
Table I. Mobility Metrics

\begin{tabular}{|c|l|}
\hline 1 & Degree of Direction AutoCorrelation \\
\hline 2 & Degree of Speed AutoCorrelation \\
\hline 3 & Entropy of Direction Probability Distribution \\
\hline 4 & $\begin{array}{l}\text { Entropy of Direction Change Probability } \\
\text { Distribution }\end{array}$ \\
\hline 5 & Entropy of Position Density Probability \\
\hline 6 & Average speed \\
\hline 7 & Average Acceleration \\
\hline 8 & Speed Change Rate \\
\hline 9 & Direction Change Rate \\
\hline 10 & Stop Rate \\
\hline 11 & Speed Variance \\
\hline
\end{tabular}

\section{RELATED WORKS}

The most related works to ours are works done on analysis the nature of human mobility such as [9], [5], [10], [11]. To the best of our knowledge the most comprehensive work in this area is [5] in which researchers have analysed probability distribution of flight length, pause time, flight speed, and mean squared displacement of human mobility. They have used the data collected from 5 different places and done statistical analysis to find the best probability distribution for each of the above features. Then they have used the results of the statistical analysis to propose a mobility model called Levywalk.

Although [5] is one of the best works done on analysis on human mobility, there are some drawbacks in the work (which we are aiming to deal with in this paper) as follows, they have used linear interpolation for estimating the missing GPS waypoint and have not considered the GPS noise and the shape of the road networks [12] and their impact on the quality of their statistical analysis. Moreover, they have not considered different transportation modes in their analysis. Furthermore, they have not analysed the discriminative power of each of the metrics they have proposed to distinguish between real world trajectories. In addition, they have not proposed a comprehensive methodology for pattern recognition and analysis of the similarity between real world trajectories and mobility models using different mobility metrics.

\section{MOBILITY METRICS}

In order to analyze mobility trajectories, we need to extract features that represent their actual motion behavior in real world environments. For extraction of these features we need to define some mobility metrics of motion trajectories. In this section we briefly introduce our mobility metrics we have used in our feature extraction method [6], [7], [13].

Table I assigns a number to each of the mobility metrics to achieve simplicity in naming them.

\section{A. Degree of Direction Autocorrelation}

This metric examines the degree of temporal dependence between a node's direction at the current sample time and $\Delta \mathrm{t}$ sample time earlier [6]. We take this time difference to be 1 sample time [6]. 


$$
\operatorname{DAC}\left(i, t, t^{\prime}\right)=\operatorname{RD}\left(\vec{v}_{i}(t), \vec{v}_{i}\left(t^{\prime}\right)\right)=\cos (\theta),
$$

where $\mathrm{RD}$ is the cosine of angle between two vectors given by:

$$
\frac{\vec{a}(t) \cdot \vec{b}(t)}{\|\vec{a}(t)\|\|\vec{b}(t)\|}
$$

The Average Degree of Direction Correlation, which is the average of Direction Correlation over all nodes and all time instances in a mobility trace, is calculated as follows [6]:

$$
\overline{\mathrm{DAC}}=\frac{\sum_{t=1}^{T} \mathrm{DAC}\left(i, t, t^{\prime}\right)}{T} .
$$

\section{B. Degree of Speed Autocorrelation}

This metric examines the degree of temporal dependence between a node's speed from the current sample time to $\Delta \mathrm{t}$ sample time earlier [6]. We take this time difference to be 1 sample time [6].

$$
\operatorname{SAC}\left(i, t, t^{\prime}\right)=\operatorname{SR}\left(\overrightarrow{s_{i}}(t), \overrightarrow{s_{i}}\left(t^{\prime}\right)\right),
$$

where $\mathrm{SR}$ is the speed ratio between two vectors, is given by

$$
\mathrm{SR}=\frac{\min \left|\overrightarrow{s_{i}}(t), \overrightarrow{s_{i}}\left(t^{\prime}\right)\right|}{\max \left|\overrightarrow{s_{i}}(t), \overrightarrow{s_{i}}\left(t^{\prime}\right)\right|}
$$

The Average Degree of Speed Auto Correlation, which is the average of Degree of Speed Auto Correlation over all time instances in a trajectory, is calculated as follows [6]:

$$
\overline{\mathrm{SAC}}=\frac{\sum_{t=1}^{T} \operatorname{SAC}\left(i, t, t^{\prime}\right)}{T},
$$

where $P$ is the number of tuples $\left(n, t, t^{\prime}\right)$.

\section{Average Speed}

The speed of a mobile node is obviously one useful feature for separation of transportation modes [13]. To extract this feature we have calculated the average of speeds which are more than a certain threshold $(0.2 \mathrm{~m} / \mathrm{s})$. We did this to eliminate the impact of zero speeds in the average speed. This feature has high value in "Car", "Train" and "Bus" and lower value in "Bike" and "Walk" [13]. 


\section{Speed Variance}

Speed variance shows the variance of speed of a mobile node during its trajectory time period [13]. This feature has high value in "Car", "Train" and "Bus" and lower value in "Bike" and "Walk" as well.

\section{E. Average Acceleration}

Acceleration variance shows the variance of speed of a mobile node during its trajectory time period. Since the acceleration can have positive and negative values we have used the absolute value of acceleration to have a better view of acceleration of our trajectories [13].

\section{F. Direction Change Rate}

Direction or heading change rate indicates how frequently a mobile node changes its heading direction during their trajectories [13]. This feature is higher in "Walk" because people are not usually as restricted to road networks (with constant direction in a straight road segment) as cars and buses are.

This value is rather higher in "Bike" as well. To calculate this feature we have found the number of the heading direction changes (DC) which are larger than a certain threshold. Then we normalize this value, for each trajectory, by dividing it by distance or length of the trajectory (in meters).

$\mathrm{DCR}=\mathrm{DC} /$ TrajectoryDistance

\section{G. Speed Change Rate}

In trajectories with transportation modes such as "Walk", "Bike" and "Bus" the the frequency of speed changes are higher than others [13]. To calculate this feature, we have considered speed changes which are more than a certain threshold as a speed change. Then we have divided the number of speed changes during a trajectory time period by length of the trajectory similar to above.

\section{H. Stop Rate}

In trajectories with transportation modes such as "Walk", "Bike" and "Bus" the frequency of stops are higher than others [13]. Therefore, this feature is a good feature to distinguish between different transportation modes. To calculate this feature, we have found the frequency of stops and divided it by the length of trajectories similar to above.

\section{Entropy of Position Probability Distribution}

Position Density measures how mobile nodes are distributed in the analysis area of each trajectory. To generate this metric we first find the location density distribution for each of the trajectories and then find the entropy of the probability distribution. This metric would be high in trajectories where mobile nodes are located in specific areas of a map, considerably more than at other areas, i.e. in nonuniform distributions, and low in traces where nodes are uniformly 
distributed [6]. For example, in the traces with bus transportation mode, since mobile nodes are restricted to streets in an urban map, most of the time their position distribution density is much higher in street areas than building blocks (compared to walk transportation mode) and similarly higher in intersections and bus stops than streets. Therefore, this metric would be appropriate to distinguish trajectories that have more geographical restrictions [6]. Note that, in our previous work, we have used variance of position densities instead of entropy which is less discriminative.

To generate the metric, first we extract position density of mobile nodes in each trajectory throughout the trajectory period, by transforming our trajectory coordination system from geodetic to Cartesian, dividing our trajectory areas into cellular areas and calculating the density of the mobile nodes in each cell inside the grid environment of each trajectory.

The Position probability in each of the cells is computed as follows:

$$
P(\operatorname{Exists}(i, j))=\frac{\sum_{t=1}^{T} \operatorname{Exists}(t, i, j)}{T},
$$

where $\operatorname{Exists}(i, j, t)=1$ if node $\mathrm{n}$ exists in Cell $(i, j)$ at time $t$, and otherwise 0 .

In other words, position probability is defined as the probability of existence of a mobile nodes in a particular cell in a specific time. Then we calculate Entropy of Position Probability Distribution as follows:

$$
H(\text { Exists })=-\sum_{\mathrm{i}=1}^{I} \sum_{\mathrm{j}=1}^{J} P(\operatorname{Density}(\mathrm{i}, \mathrm{j})) \log _{2}(P(\operatorname{Density}(\mathrm{i}, \mathrm{j}))),
$$

where $i$ and $j$ are coordinates of cells in the simulation area. In our analysis $I$ and $J$ are set according to width and height of our trajectory environment

\section{J. Entropy of Direction Change Probability Distribution}

This metric allows us to compare different types of transportation modes. It shows the degree of uniformity in the direction changes of a mobile node during its trajectory period. In an urban area, vehicles and people are restricted to streets and road networks. The turning angles of vehicles are restricted mostly to some particular angles. For instance, in urban areas, objects are not able to turn and change their directions with a random angle. They are restricted to turn based on the road network conditions. In contrast, an animal in a farm is often able to move almost in any direction or turn with any angle. Therefore, density of direction changes is substantially non-uniform in urban areas in contrast areas with no geographical restrictions.

To extract direction change probability distribution of a trajectory, we use our method propose in [7]. Firstly, we calculated direction changes of the moving object in each of the trajectories. Then we calculated the probability of each of the direction change angles between 1 and 180 degrees. Thus, we generated a list of 180 direction change angles and their probabilities. We have rounded the direction changes to have a discrete set of direction changes $\{1 ; 2 ;::: ; 180\}$. Probability for 
direction change with angle $\beta$ is defined as ${ }^{2} \mathrm{P}\left(\mathrm{DC}_{\beta}\right)=\frac{D C_{\beta}}{{ }^{A}} \cdot \mathrm{DC}_{\beta}$ is the count of direction changes with angle ${ }^{\beta}$ and $\mathrm{A}$ is the count of all direction changes in the trajectory [7].

We propose Entropy of Direction Change probability Distribution as a numeric measure to compare direction change probability distributions of trajectories with different transportation modes [7]. In information theory and probability, entropy [14] is a measure of amount of information in a signal. It indicates amount of disorder and degree of uncertainty of a probability distribution as well. Shannon's Entropy of direction change probability distribution of a trajectory is defined as follows [7]:

$$
H(\mathrm{DC})=-\sum_{\alpha=1}^{180} P\left(\mathrm{DC}_{\alpha}\right) \log _{2}\left(P\left(\mathrm{DC}_{\alpha}\right)\right)
$$

\section{K. Entropy of Direction Probability Distribution}

In urban environments in accordance with the shape of the map of road networks, vehicles and people usually move with some particular directions much more than others. As a result the probability distribution of direction angles of moving objects is not uniform. Degree of uniformity or degree of disorder of direction probability distribution can be considered as another measure for comparison of trajectories with different transportation modes. To be able to analyze the behavior of our trajectories, we calculated the entropy of direction probability distribution of trajectories, similar to entropy of direction change probability distribution [7] discussed in III-J with the difference that here we have 360 rounded discrete direction angles $\{1 ; 2 ;:: ; 360\}$. We define Shannon's Entropy of direction change probability distribution of a trajectory as follows [7]:

$$
H(\mathrm{DA})=-\sum_{\alpha=1}^{360} P\left(\mathrm{DA}_{\alpha}\right) \log _{2}\left(P\left(\mathrm{DA}_{\alpha}\right)\right) .
$$

\section{SIMILARITY ANALYSIS}

In this section we briefly introduce the steps of our similarity analysis methodology. Our purpose is to find the degree of similarity between each of the real world transportation modes to each of our mobility models. Aiming for that, we first pre-process our real world trajectories to make them suitable for feature extraction (IV-A). Secondly, we find the best feature sets which are able to discriminate and distinguish different transportation modes from each other (IV-B) using a supervised learning method. Subsequently, using the best feature sets we perform the Fuzzy Cmeans clustering method to find the degree of membership of each of the transportation modes to each of our mobility model clusters and consider this membership as degree of similarity between mobility models and transportation modes (IV-D).

As mentioned before, selection of the similarity metrics is a major issue in any similarity analysis because the degree of similarity is highly dependent on the feature space we are using in our machine learning process [15]. We use the best feature set selected in the feature selection phase discussed in IV-B as the feature space in the FCM method because we have found that it is the 
optimum feature set that can distinguish trajectories with different transportation modes from each other with maximum accuracy.

\section{A. Trajectory Pre-processing}

We have used a very large database of trajectories collected by Microsoft Research in Beijing, China [16], [17], This dataset contains trajectories of 181 people moving for almost 3 years. We inserted every trajectory with known transportation mode into our database. Then, we selected 100 trajectories (with highest GPS sampling rate) of each of the transportation modes. Hence we have a dataset of trajectories of different mobile nodes with known transportation modes.

To be able to extract the metrics introduced above, we needed to process our raw GPS trajectories to make them suitable for feature extraction. Due to irregularity and low ampling rate in trajectories collected by GPS, we do not have access to data about position, speed and direction of our mobile nodes at all needed time samples. For example, in our processing we need to compute the direction autocorrelation of each mobile node once every 10 seconds. However, the GPS trajectories sampling rate is not regular and do not include spatio-temporal information of the mobile nodes regularly for all needed times. Moreover, particularly in urban environments we need to provide map-matching [12], [18], [19] to reduce GPS noise and make our trajectories more dependable. In previous related works such as [5], linear interpolation has been applied on the raw GPS trajectories to interpolate missing waypoints (at needed sampling times). However, linear interpolation extremely suffers from inaccuracy [7].

We have used our previously proposed method called map based spatio-temporal interpolation [7] to interpolate missing waypoints and do map-matching on our trajectories. Map based interpolation uses real world maps and the estimated speed for each road segment to estimate the position of each mobile node at each given query time on the road network. We have called HSTQ query [7] for each needed sampling time and up-sampled our real world trajectories to archive regularly sampled GPS trajectories with reduced GPS noise. As opposed to linear interpolation used in [5], map-based interpolation has very higher accuracy in estimated positions and estimated turning angles and speeds. This method implicitly does mapmatching [12], [20] to reduce GPS noise as well.

After performing the pre-processing phase, we have 100 trajectories for each of our transportation modes ("Walk", "Car", "Bike", "Train", "Bus") extracted from our dataset [16] with regular sampling rate and reduced GPS noise suitable enough for our feature extraction phase. Then we separated the trajectories into two sets of training and test trajectories for each of the transportation modes.

\section{B. Classification and Feature Selection}

The goal of the feature selection [15] process is to find the best (most discriminative) feature subsets (comprised of calculated values of mobility metrics) which are able to distinguish between each specific transportation mode from other transportation modes. The feature set should be optimal. In other words, we should find feature sets with the highest accuracy and minimum set cardinality. For example if we can use only speed Autocorrelation mobility metric to distinguish between "Walk" and "Car" transportation modes, it does not make sense to run the feature extraction and classification using all the mobility metrics. Therefore, we choose the most 
discriminative feature set as our similarity analysis feature set among so many different possible feature sets (in our study it would be $2^{11}=2048$ different feature sets) [6].

Depending on our trajectories and our environment conditions (shape of the road network and traffic conditions, etc), the best feature set selected by our feature selection method may slightly change. Therefore, we should perform all the steps proposed in this paper to find the best feature set and find the degree of similarity between our mobility models and real world trajectories in that particular environment.

Table II. Selected Optimal Feature Sets

\begin{tabular}{|c|c|c|}
\hline Transportation Mode & Feature Sets & Accuracy (\%) \\
\hline All & $\{1,3,4,9\}$ & $74.4 \%$ \\
\hline Walk & $\{7,8,9\}$ & $74 \%$ \\
\hline Car & $\{11\}$ & $98 \%$ \\
\hline Bus & $\{1,6,11\}$ & $48 \%$ \\
\hline Bike & $\{1,3,4,8,10\}$ & $78 \%$ \\
\hline Train & $\{7,11\}$ & $98 \%$ \\
\hline
\end{tabular}

1) Feature Extraction: We performed the feature extraction phase resulting in two training and test tables each of which include 250 rows and 11 columns (mobility metrics in tableI). We also generated test tables for each of the transportation modes separately (each including 50 rows) to be able to analyse performance of our feature sets for each of the transportation modes separately.

2) Classification: We used the K-nearest neighbor $(\mathrm{KNN})$ [15] classification method to classify each of our samples in the test table to it's nearest classes. We trained the classifier with our labeled training samples. Then we classify the test samples into their nearest classes. Euclidian distance was used for finding $k$ nearest neighbors and parameter $\mathrm{K}$ was set to 10 [6].

3) Feature Ranking: To find the optimal feature set, firstly, we generated all possible feature subsets of our feature set. Subsequently, we tested the accuracy of each of the feature subsets in classification of different transportation modes. Then we ranked each feature set using its classification accuracy combined with cardinality of feature set [6].

4) Results: Table II shows selected most discriminative and best feature sets for classification of each of transportation mode. The word "All", means we have used every 250 test trajectories for classification. As it is clearly seen, the best feature set which is able to distinguish all the transportation modes from each other with highest accuracy $(74: 4 \%)$ and lowest set cardinality (4) is $\{1 ; 3 ; 4 ; 9\}$. Suppose we have a trajectory with unknown transportation mode, our experimental results suggest that if we need to classify it into its right transportation mode class we should use features $\{1 ; 3 ; 4 ; 9\}$ (feature space comprised of degree of direction autocorrelation, entropy of direction probability distribution, entropy of direction change probability distribution, and direction change rate) and make a 4 dimensional feature space for our classification.

Based on results depicted in table II, for example, if we need to classify trajectories with "Walk" transportation mode into right class ("Walk" class) we should use feature set comprised of speed change rate and direction change rate features. 
By using this feature set if a trajectory had a "Walk" transportation mode, it will be detected with $98 \%$ accuracy and there is $2 \%$ chance for it being classified wrongfully. We can infer the same results from other rows in table II similarly.

\section{Mobility Models Simulation Configurations}

To be able to preform similarity analysis using the selected feature set, $(\{1 ; 3 ; 4 ; 9\})$, we generated one mobility trace for each of the following mobility models that we needed to analyse: (RW, RWP, RPGM, MAN, and LW) using our previously developed mobility simulator [2]. Each of the mobility traces include 50 mobile nodes moving in an area of $10 \mathrm{~K} \mathrm{X} \mathrm{10K} \mathrm{meters} \mathrm{moving}$ for 10000 sample times. Minimum and maximum speed in all the models are set as $1 \mathrm{~m} / \mathrm{s}$ and 25 $\mathrm{m} / \mathrm{s}$ respectively. Maximum pause times for RWP and Manhattan have set to 10 sample times and minimum pause time to 1 sample time. We used a regular Manhattan map for the Manhattan mobility model with 50 equidistant intersections in the simulation area [2]. For Levy Walk mobility model, we set the maximum pause time to 10 and minimum pause time to 1 sample times. We set arameters $\alpha$ to 1 , max flight to 100 meter, min flight 30 meter and parameter ${ }^{\beta}$ to 1 [5]. For RPGM mobility model, the central node moves with RW model. The $s d r$ and $a d r$ parameters in RPGM have been set to 0:05. Walk time parameter in RW has been set to 20 sample times.

Then we performed the feature extraction similar to the way discussed in IV-B1.

\section{Fuzzy C-means Clustering}

To perform similarity analysis we use Fuzzy C-means clustering algorithm [8]. FCM is a clustering method which in addition to estimating the cluster to which a sample belongs, reports the degree of membership of a sample to all the clusters. We use the reported memberships ([0; 1]) converted to percentage as a measure of similarity of a sample to a cluster.

We generated a table comprising of 250 rows and 11 column (metrics). We also used the same (real world) trajectories used as the training set in IV-B. For simplicity, we used the average of features extracted form each of the group of the transportation modes as the representative of each of the transportation modes. So, for each of the transportation modes we had a new table with 251 rows. The last row contains average value for each metric for the particular transportation mode. We used the FCM method using MATLAB on our extracted data. The number of desired clusters in FCM has been set to 5 (the count of the transportation modes).

Table III shows the results provided by FCM clustering method with the configurations discussed above. It shows the degree of similarity between each of the transportation modes and each of our considered mobility models (in percent).

Although the purpose of this paper is to propose a comprehensive similarity analysis ethodology, not to find the best mobility models for simulation, one interesting outcome of our experimental results shows that Levy Walk [5] is the best mobility model among our mobility models in four of the transportation modes. This similar results to [5] can confirm the performance of our similarity measurement method and also confirm the performance of LW mobility model to mimic the real world mobility using new mobility models and more comprehensive analysis. On the other hand, the Random Waypoint mobility model, although being one of the most highly used mobility 
models used in mobile networks simulations such as DTN and MANET protocols [3], [4], has much lower performance in comparison with Levy Walk; based on our experiments using our selected features. Random walk which is very similar to Brownian motion also does not perform well in most cases.

Table III. Degree of Similarity

\begin{tabular}{|c|c|c|}
\hline Transportation Mode & Mobility Model & Degree of Similarity (\%) \\
\hline \multirow{5}{*}{ Car } & RW & $54.25 \%$ \\
\hline & LW & $36.84 \%$ \\
\hline & RWP & $4.35 \%$ \\
\hline & RPGM & $2.62 \%$ \\
\hline & MAN & $1.75 \%$ \\
\hline \multirow{5}{*}{ Bike } & $\overline{\mathrm{LW}}$ & $43.34 \%$ \\
\hline & RW & $29.69 \%$ \\
\hline & RWP & $11.80 \%$ \\
\hline & RPGM & $9.65 \%$ \\
\hline & MAN & $5.52 \%$ \\
\hline \multirow{5}{*}{ Bus } & $\overline{\mathrm{LW}}$ & $56.66 \%$ \\
\hline & RWP & $22.51 \%$ \\
\hline & RW & $11.81 \%$ \\
\hline & MAN & $5.09 \%$ \\
\hline & RPGM & $3.93 \%$ \\
\hline \multirow{5}{*}{ Train } & LW & $62.26 \%$ \\
\hline & RW & $27.72 \%$ \\
\hline & RWP & $5.60 \%$ \\
\hline & RPGM & $2.44 \%$ \\
\hline & MAN & $1.98 \%$ \\
\hline \multirow{5}{*}{ Walk } & $\overline{\mathrm{LW}}$ & $33.87 \%$ \\
\hline & RW & $23.88 \%$ \\
\hline & RWP & $18.51 \%$ \\
\hline & RPGM & $13.31 \%$ \\
\hline & MAN & $10.43 \%$ \\
\hline
\end{tabular}

\section{CONCLUSION AND FUTURE WORK}

In this paper we proposed a comprehensive methodology for measurement of similarity between mobility models often used in simulation of mobile networks protocols and real world motion trajectories with different transportation modes. We introduced metrics and a method for classification of trajectories with different transportation modes. Subsequently, we briefly introduced a pre-processing method we need to perform to achieve GPS trajectories with regular sampling rate and reduced noise. Then we used a feature ranking method and found best features for classification of each of the transportation modes from others. Then we provided a method for similarity analysis using Fuzzy C-means clustering method. Depending on our trajectories and environment of our networking protocol, the best feature set selected by our feature selection method may change. Hence, we need to perform all the steps proposed in this paper to find the best feature set and find the degree of similarity between our mobility models (which can be 
different to the ones we have considered) and real world trajectories (with other kinds of transportation modes in different area with other road networks and traffic conditions).

As future work, we will consider new features such as frequent and periodic behaviour to distinguish between similar transportation modes such as cars and buses using time series similarity analysis. Moreover, we will work on proposing mobility models which are able to simulate frequent and periodic behaviours other than spatio-temporal, geographical behaviours.

\section{REFERENCES}

[1] S. Ioannidis and P. Marbach, "A brownian motion model for last encounter routing." in INFOCOM. IEEE. [Online]. Available: http://dblp.uni-trier.de/db/conf/infocom/infocom2006.htm

[2] S. M. Mousavi, M. Moshref, H. R. Rabiee, and A. Dabirmoghaddam, "Mobisim: a framework for simulation of mobility models in mobile ad-hoc networks," in Proceedings of 3rd IEEE International Conference on Wireless and Mobile Computing, Networking and Communications. New York, USA, October 2007.

[3] F. Bai, N. Sadagopanb, and A. Helmy, "The important framework for analyzing the impact of mobility on performance of routing protocols for adhoc networks," Ad Hoc Networks, vol. 1, no. 4, pp. 383-403, November 2003.

[4] T. Camp, J. Boleng, and V. Davis, "A survey of mobility models for ad hoc network research," Wireless Communication and Mobile Computing, vol. 2, no. 5, pp. 483-502, August 2002.

[5] I. Rhee, M . Shin, S. Hong, K. Lee, and S. Chong, "On the levy-walk nature of human mobility," Networking, IEEE/ACM Transactions on, vol. 19, no. 3, pp. 630-643, June 2011.

[6] M. M. Barroudi, A. Harwood, and S. Karunasekera, "Feature selection for user motion pattern recognition in mobile networks," in PIMRC, 2012, pp. 1521-1527.

[7] _ - "Map-based spatio-temporal interpolation in vehicle trajectory data using routing webservices," in Proceedings of the 5th ACM SIGSPATIAL International Workshop on Computational Transportation Science, ser. IWCTS '12. New York, NY, USA: ACM, 2012, pp. 43-48. [Online]. Available: http://doi.acm.org/10.1145/2442942.2442951

[8] J. Bezdek, R. Ehrlich, and W. Full, "FCM: The fuzzy c-means clustering algorithm," Computers \& Geosciences, vol. 10, no. 2-3, pp. 191-203. [Online]. Available: http://dx.doi.org/10.1016/00983004(84)90020-7

[9] M. C. Gonzalez, C. A. Hidalgo, and A.-L. Barabasi, "Understanding individual human mobility patterns," Nature, vol. 453, no. 7196, pp. 779-782, Jun. 2008. [Online]. Available: http://dx.doi.org/10.1038/nature06958

[10] K. Lee, S. Hong, S. J. Kim, I. Rhee, and S. Chong, "Slaw: Self-similar least-action human walk," IEEE/ACM Trans. Netw., vol. 20, no. 2, pp. 515-529, Apr. 2012. [Online]. Available: http://dx.doi.org/10.1109/TNET.2011.2172984

[11] D. Brockmann, L. Hufnagel, and T. Geisel. (2006, May) The scaling laws of human travel. [Online]. Available: http://arxiv.org/abs/condmat/0605511

[12] M. A. Quddus, W. Y. Ochieng, and R. B. Noland, "Current mapmatchingalgorithms for transport applications: State-of-the art and future research directions," Transportation Research Part C: Emerging Technologies, vol. 15, no. 5, pp. 312 - 328, 2007. [Online]. Available: http://www.sciencedirect.com/science/article/pii/S0968090X07000265

[13] Y. Zheng, Q. Li, Y. Chen, X. Xie, and W.-Y. Ma, "Understanding mobility based on gps data," in Proceedings of the 10th international conference on Ubiquitous computing, ser. UbiComp '08. New York, NY, USA: ACM, 2008, pp. 312-321. [Online]. Available: http://doi.acm.org/10.1145/1409635.1409677

[14] A. Papoulis, Probability, Random Variables, and Stochastic Processes, 4th ed., 2002.

[15] R. O. Duda, P. E. Hart, and D. G. Stork, Pattern classification. Wiley,2001.

[16] Y. Zheng, X. Xie, and W.-Y. Ma, "Geolife: A collaborative social networking service among user, location and trajectory," IEEE Data Eng.Bull., vol. 33, no. 2, pp. 32-39, 2010. 
[17] —, "Mining interesting locations and travel sequences from gps trajectories," in WWW 2009. Association for Computing Machinery, Inc., April 2009, wWW 2009. [Online]. Available:http://research.microsoft.com/apps/pubs/default.aspx?id=79440

[18] Y. Zheng and X. Zhou, Eds., Computing with Spatial Trajectories.Springer, $2011 .$.

[19] G. Trajcevski, "Uncertainty in spatial trajectories," in Computing with Spatial Trajectories, 2011, pp. 63-107.

[20] J. Krumm, "Trajectory analysis for driving," in Computing with Spatial Trajectories, 2011, pp. 213241. 